\title{
Synthesis and reactions of methyl 2-[3-(2-phenylquinazolin-4-yl)thioureido]alkanoates
}

\author{
Walid Fathalla ${ }^{a}$ and Pavel Pazdera ${ }^{b}$ \\ ${ }^{a}$ Department of Mathematical and Physical Sciences, Faculty of Engineering, \\ Suez Canal University, Port-Said, Egypt \\ ${ }^{b}$ Department of Organic Chemistry, Faculty of Science, Masaryk University, \\ Brno, Czech Republic \\ E-mail:walid399@yahoo.com
}

\begin{abstract}
Quinazoline thioureas 5 bearing an amino acid ester residue were prepared by a novel three step sequential reaction of $N$-(2-cyanophenyl)benzimidoyl isothioyanate with amino acid methyl ester hydrochlorides. Some chemoselective reactions of $\mathbf{5 a}$ with alkyl halides were studied.
\end{abstract}

Keywords: Non-nucleoside RT inhibitors, quinazoline, thioureas, amino acids, imidoyl isothiocyanate

\section{Introduction}

Considerable attention has been directed to the design and synthesis of new AIDS therapies. Inhibition of the viral enzyme reverse transcriptase (RT) represents particularly attractive strategy for the anti AIDS drug design. Several heterocyclic thioureas have been reported as a new class of potent non-nucleoside inhibitors of human immunodeficiency virus type 1 reverse transcriptase (NNRTIs) such phenethylthiazolyl-thiourea (PETT) derivatives. ${ }^{1-4}$

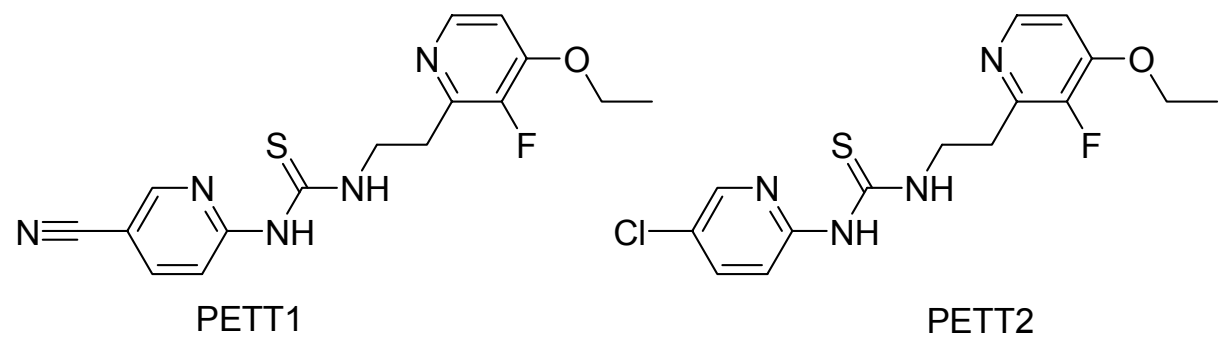

Uckun et al. ${ }^{5-7}$ described the synthesis of a number of heterocyclic thioureas having an amino acid ester or aryl side chain as NNRTIs. 


\section{Result and Discussion}

Recently, we have reported convenient one-pot syntheses of quinazolin-4-ylidenethioureas ${ }^{8}$ and quinazolin-4-ylthioureas ${ }^{9,10}$ from $N$-(2-cyanophenyl)benzimidoyl isothiocyanate with amines. $\mathrm{N}$-(2-cyanophenyl)benzimidoyl isothiocyanate $(\mathbf{1})^{8,9}$ is a highly reactive compound featuring two electrophilic sites, the nitrile and isothiocyanate functional groups in a conjugated system.

The reaction of imidoyl isothiocyanate $\mathbf{1}$ with one equivalent of amino acid ester hydrochlorides $\mathbf{2}$ in the presence of triethyl amine furnished the desired quinazolinethioureas $\mathbf{5}$ in good yields. The reaction is assumed to proceed via three steps (Scheme 1).

The amino acid ester $\mathbf{2}$ adds to the isothiocyanate function of $\mathbf{1}$ to give the open chain thiourea intermediate $\mathbf{3}$, which in turn, cyclizes by intramolecular addition of $\mathrm{NH}$ to the nitrile group giving rise to the 4-iminoquinazoline intermediate 4; a Dimroth rearrangement furnishes the isolated product, methyl 2-[3-(2-phenylquinazolin-4-yl)thioureido]alkanoate 5 (Scheme 1).

This method has the advantage of a one pot reaction with an overall moderate to good yield of 5 at room temperature to minimize the degree of racemization in amino acid coupling. The alternative thiocarbonyldiimidazole $\operatorname{method}^{5-7}$ requires a higher temperature and longer reaction time $\left(100{ }^{\circ} \mathrm{C}, 30 \mathrm{~h}\right)$ for attaching the amino acid residue at heterocyclic ring moieties.

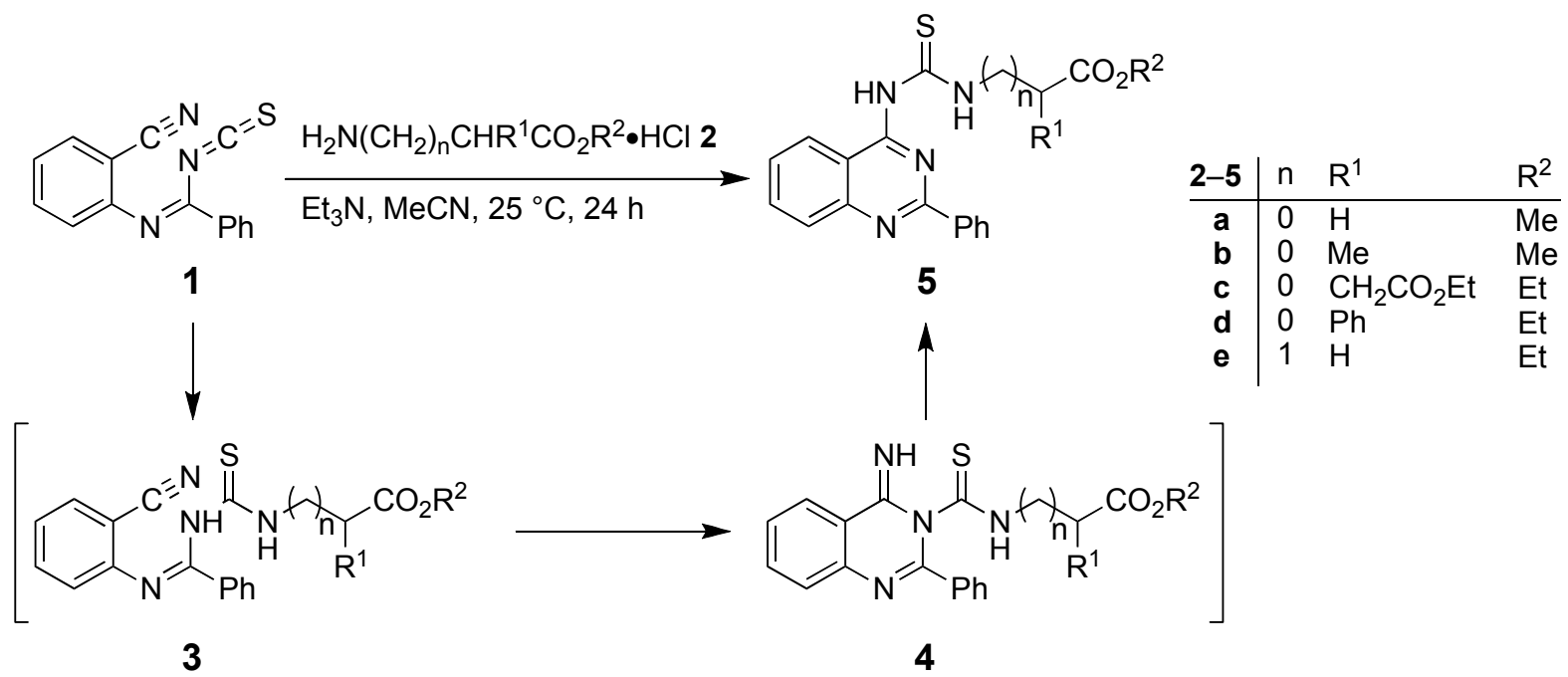

\section{Scheme 1}

The structure assignment of the amino acid derivatives $\mathbf{5 a}-\mathbf{e}$ is based on spectroscopic methods and on the correlation with a fully analyzed reference, 1-benzyl-3-(2-phenylquinazolin4-yl)thiourea ${ }^{9}$ (Figure 1). The ${ }^{1} \mathrm{H}$ NMR spectrum of 5a exhibits two signals at $\delta 12.35$ and 8.98 corresponding to $1-\mathrm{NH}$ and $3-\mathrm{NH}$ of the thiourea moiety, respectively. The ${ }^{13} \mathrm{C}$ NMR spectrum of 5a reveals quaternary carbon signals at $\delta 180.65,169.19,159.11$ and 155.90 assigned to $\mathrm{C}=\mathrm{S}$, $\mathrm{C}=\mathrm{O}, \mathrm{C} 2$ and $\mathrm{C} 4$, respectively. 


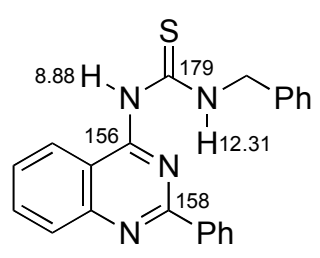

Figure 1. Selected ${ }^{1} \mathrm{H}$ and ${ }^{13} \mathrm{C}$ NMR data of 1-benzyl-3-(2-phenylquinazolin-4-yl)thiourea. ${ }^{9}$

Thioureas 5 were the only tautomers isolated, in good agreement with previous results with quinazolinethioureas bearing alkyl, aryl and heterocyclic substituents, ${ }^{9,10}$ and those reported for phenethylthiazolylthioureas. $^{5-7}$ The reaction of methyl 2-[3-(2-phenylquinazolin-4-yl)thioureido]acetate (5a) with absolute ethanol in the presence of potassium hydroxide furnished the transesterification product $\mathbf{5 f}$ (Scheme 2).

With methyl iodide, 5a underwent chemoselective $S$-alkylation to form isothiourea $\mathbf{6}^{13}$ (Scheme 2). The ${ }^{1} \mathrm{H}$ NMR spectrum of isothiourea derivative 6 shows signals at $\delta 3.5$ and 12.6, characteristic for $\mathrm{SCH}_{3}$ and $\mathrm{C}=\mathrm{N} 3 \cdots \mathrm{HN}$, respectively. According to ${ }^{1} \mathrm{H} \mathrm{NMR}$ data, isothiourea 6 is formed as a single tautomer due to hydrogen bonding.

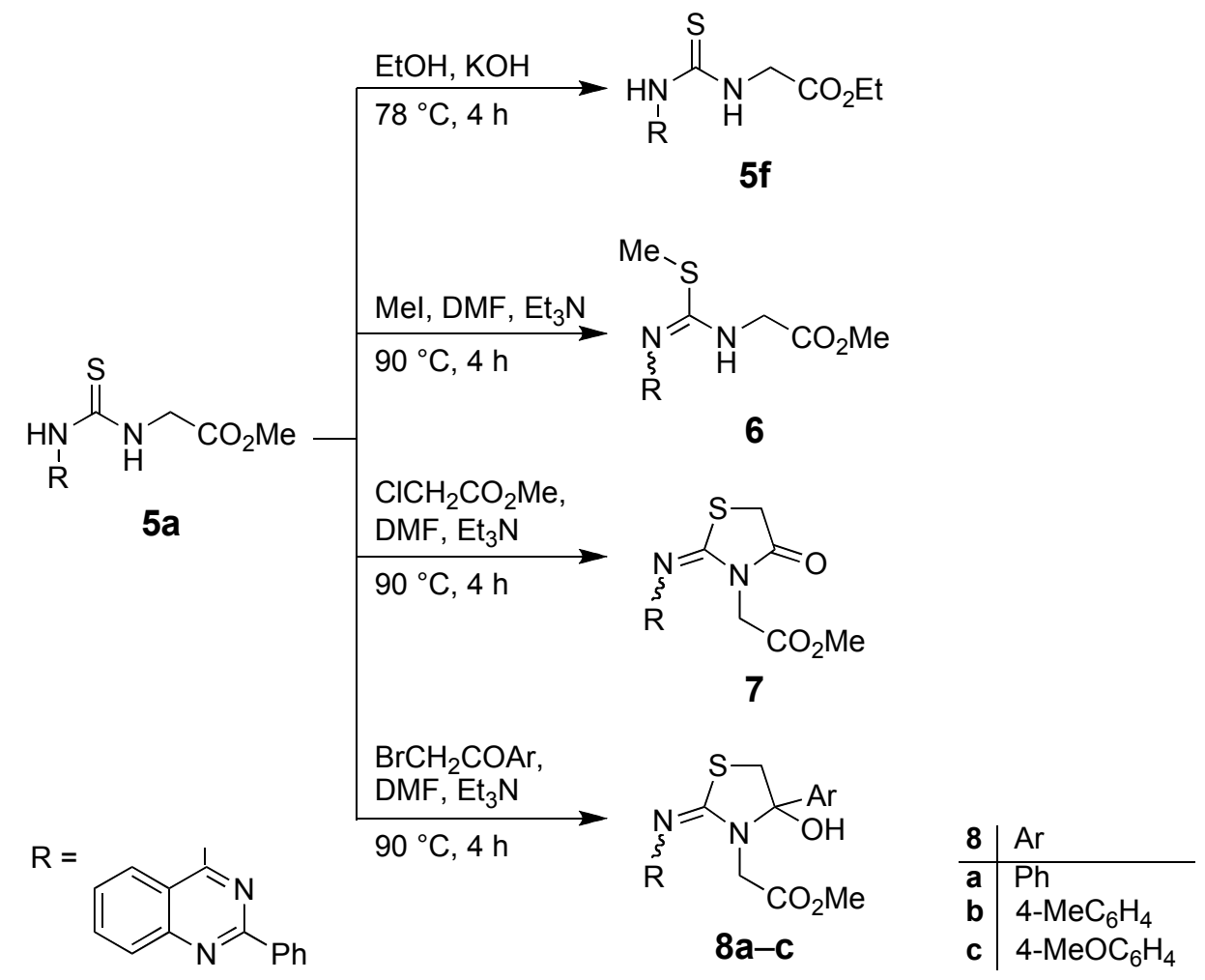

\section{Scheme 2}

Similarly, the reaction of methyl 2-[3-(2-phenylquinazolin-4-yl)thioureido]acetate (5a) with methyl chloroacetate resulted in the formation of thiazolidine 7 . The ${ }^{1} \mathrm{H}$ NMR spectrum of 7 
shows a singlet at $\delta 3.99$, the ${ }^{13} \mathrm{C}$ NMR displays a signal at $\delta 34.19$, typically associated with an $\mathrm{SCH}_{2} \mathrm{CO}$ moiety.

Following the classical Hantzsch thiazole synthesis ${ }^{14}$ the reaction of the glycinethioamide derivative 5a with phenacyl halides afforded 4-hydroxythiazolidines 8a-c. The ${ }^{1} \mathrm{H}$ NMR spectrum of compound 8a shows two doublets at $\delta 4.38$ and $\delta 3.60$ for the A parts of the AB quartets of $\mathrm{NCH}_{2}$ and $\mathrm{SCH}_{2}$, respectively. In addition, an apparent doublet is displayed at $\delta 3.49$ resulting from superimposed $\mathrm{B}$ doublets of both $\mathrm{AB}$ spectra of the $\mathrm{NCH}_{2}$ and $\mathrm{SCH}_{2}$ groups. Similarly, 8b gives rise to superimposed $\mathrm{B}$ doublets of the $\mathrm{NCH}_{2}$ and $\mathrm{SCH}_{2}$ groups. On the other hand, the $\mathrm{AB}$ doublets of $\mathrm{NCH}_{2}$ and $\mathrm{SCH}_{2}$ in $\mathbf{8 c}$ are well separated at $\delta 4.39,3.58$ and $\delta 3.82$, 3.49 , respectively.

\section{Conclusions}

The reaction of $N$-(2-cyanophenyl)benzimidoyl isothiocyanate (1) with amino acid esters 2 afforded methyl 2-[3-(2-phenylquinazolin-4-yl)thioureido]alkanoates (5) via a three step sequential reaction. Compound 5a underwent alkylation reactions with methyl chloroacetate and phenacyl halides to afford thiazolidine derivatives 7 and $\mathbf{8 a}-\mathbf{c}$, respectively.

\section{Experimental Section}

General Procedures. Melting points were measured on a Boetius Rapido PHMK 79/2106 (Wägetechnik) instrument. Elemental analysis were carried out with an Erba 1102 instrument. ${ }^{1} \mathrm{H}$ and ${ }^{13} \mathrm{C}$ NMR spectra were recorded at $300 \mathrm{MHz}$ and $75.5 \mathrm{MHz}$, respectively (DRX 500 Avance Bruker) in $\mathrm{CDCl}_{3}$ solution with tetramethylsilane as an internal standard.

Methyl 2-[3-(2-phenylquinazolin-4-yl)thioureido]alkanoates (5). General Method. To a solution of amino acid ester hydrochloride $2(5 \mathrm{mmol})$ in acetonitrile $(10 \mathrm{~mL})$ was added triethyl amine $(0.7 \mathrm{~mL}, 5 \mathrm{mmol})$. This solution was stirred at $5{ }^{\circ} \mathrm{C}$ for $30 \mathrm{~min}$, filtered and subsequently added in portions to a previously prepared solution of imidoyl isothiocyanate $\mathbf{1}(1.32 \mathrm{~g}, 5 \mathrm{mmol})$ in acetonitrile $(30 \mathrm{~mL}) .{ }^{8,9}$ The reaction mixture was stirred at room temperature for $24 \mathrm{~h}$. The solvent was evaporated under reduced pressure, and the residue was crystallized from ethanol.

Methyl 2-[3-(2-phenylquinazolin-4-yl)thioureido]acetate (5a). From $\mathrm{GlyOCH}_{3} \cdot \mathrm{HCl} \mathbf{2 a}(0.63$ g). Colorless crystals (1.23 g, 69\%); mp 197-198 ${ }^{\circ} \mathrm{C} .{ }^{1} \mathrm{H}$ NMR $\left(300 \mathrm{MHz}, \mathrm{CDCl}_{3}\right): \delta 12.35(1 \mathrm{H}$, s, $\left.\mathrm{NHCH}_{2}\right), 8.98$ (1H, s, 4-NH), 8.54-8.51 (2H, m, ArH), 8.04-8.01 (1H, m, ArH), 7.93-7.88 $\left.(2 \mathrm{H}, \mathrm{m}, \mathrm{ArH}), 7.64-7.56(4 \mathrm{H}, \mathrm{m}, \mathrm{ArH}), 4.68\left(2 \mathrm{H}, \mathrm{d}, J=4.66 \mathrm{~Hz}, \mathrm{NHC}_{2}\right) 4.7 \mathrm{~Hz}\right), 3.85(3 \mathrm{H}, \mathrm{s}$, $\left.\mathrm{OCH}_{3}\right) ;{ }^{13} \mathrm{C} \mathrm{NMR}\left(75.5 \mathrm{MHz}, \mathrm{CDCl}_{3}\right): \delta 180.65(\mathrm{C}=\mathrm{S}), 169.19(\mathrm{C}=\mathrm{O}), 159.11(\mathrm{C}=\mathrm{N}, \mathrm{C} 2)$, $155.90(\mathrm{C}=\mathrm{N}, \mathrm{C} 4), 151.54\left(\mathrm{C}_{\mathrm{qAr}}\right), 137.31\left(\mathrm{C}_{\mathrm{qAr}}\right), 134.58\left(\mathrm{CH}_{\mathrm{Ar}}\right), 131.12\left(\mathrm{CH}_{\mathrm{Ar}}\right), 129.84\left(\mathrm{CH}_{\mathrm{Ar}}\right)$, $128.68\left(\mathrm{CH}_{\mathrm{Ar}}\right), 127.79\left(\mathrm{CH}_{\mathrm{Ar}}\right), 120.60\left(\mathrm{CH}_{\mathrm{Ar}}\right), 112.58\left(\mathrm{C}_{\mathrm{qAr}}\right), 52.75\left(\mathrm{OCH}_{3}\right), 48.27\left(\mathrm{NCH}_{2}\right)$. 
Anal. Calcd. for $\mathrm{C}_{18} \mathrm{H}_{16} \mathrm{~N}_{4} \mathrm{O}_{2} \mathrm{~S}$ (352.4): C, 61.35; H, 4.58; N, 15.90; S, 9.10. Found: C, 61.23; H, $4.49 ; \mathrm{N}, 15.88 ; \mathrm{S}, 8.96$.

Methyl 2-[3-(2-phenylquinazolin-4-yl)thioureido]propanoate (5b). From $\mathrm{L}-\mathrm{AlaOCH} \cdot \mathrm{HCl}_{3} \mathbf{2 b}$ (0.7 g). White crystals $(0.89 \mathrm{~g}, 49 \%)$; mp 182-183 ${ }^{\circ} \mathrm{C} .{ }^{1} \mathrm{H} \mathrm{NMR} \quad\left(300 \mathrm{MHz}, \mathrm{CDCl}_{3}\right): \delta 12.34$ $\left(1 \mathrm{H}, \mathrm{s}, \mathrm{NHCH}_{2}\right), 8.87$ (1H, s, 4-NH), 8.53-8.51 (2H, m, ArH), 8.03-7.98 (1H, m, ArH), 7.937.89 (2H, m, ArH), 7.64-7.55 (4H, m, ArH), 5.45-5.39 (1H, m, CH), $3.87\left(3 \mathrm{H}, \mathrm{s}, \mathrm{OCH}_{3}\right), 1.35$ $\left(3 \mathrm{H}, \mathrm{d}, J=6.9 \mathrm{~Hz} \mathrm{CHCH}_{3}\right) ;{ }^{13} \mathrm{C} \operatorname{NMR}\left(75.5 \mathrm{MHz}, \mathrm{CDCl}_{3}\right): \delta 180.13(\mathrm{C}=\mathrm{S}), 172.58(\mathrm{C}=\mathrm{O})$, $159.01(\mathrm{C}=\mathrm{N}, \mathrm{C} 2), 155.98(\mathrm{C}=\mathrm{N}, \mathrm{C} 4), 151.58\left(\mathrm{C}_{\mathrm{qAr}}\right), 137.45\left(\mathrm{C}_{\mathrm{qAr}}\right), 134.59\left(\mathrm{CH}_{\mathrm{Ar}}\right), 131.17$ $\left(\mathrm{CH}_{\mathrm{Ar}}\right), 129.85\left(\mathrm{CH}_{\mathrm{Ar}}\right), 128.89\left(\mathrm{CH}_{\mathrm{Ar}}\right), 128.67\left(\mathrm{CH}_{\mathrm{Ar}}\right), 127.83\left(\mathrm{CH}_{\mathrm{Ar}}\right), 120.61\left(\mathrm{CH}_{\mathrm{Ar}}\right), 112.63$ $\left(\mathrm{C}_{\mathrm{qAr}}\right), 54.84\left(\mathrm{OCH}_{3}\right), 52.81\left(\underline{\mathrm{CHCH}}_{3}\right), 18.37\left(\mathrm{CHCH}_{3}\right)$. Anal. Calcd. For $\mathrm{C}_{19} \mathrm{H}_{18} \mathrm{~N}_{4} \mathrm{O}_{2} \mathrm{~S}(366.4)$ : C, 62.28; H, 4.95; N, 15.29; S, 8.75. Found: C, 62.27; H, 4.95; N, 15.24; S, 8.73.

Diethyl 2-[3-(2-phenylquinazolin-4-yl)thioureido]succinate (5c). From DL-AspOC ${ }_{2} \mathrm{H}_{5} \cdot \mathrm{HCl}$ 2c (1.13 g). White crystals (1.61 g, 71\%); mp 148-149 ${ }^{\circ} \mathrm{C} .{ }^{1} \mathrm{H}$ NMR $\left(300 \mathrm{MHz}, \mathrm{CDCl}_{3}\right): \delta 12.68$ $\left(1 \mathrm{H}, \mathrm{d}, J=4.8 \mathrm{~Hz}, \mathrm{NHCH}_{2}\right), 8.90(1 \mathrm{H}, \mathrm{s}, 4-\mathrm{NH}), 8.59-8.57(2 \mathrm{H}, \mathrm{m}, \mathrm{ArH}), 8.07-8.04$ (1H, m, ArH), 7.95-7.90 (2H, m, ArH), 7.67-7.53 (4H, m, ArH), 5.54-5.67 (1H, m, CH), 4.34-4.21(2H, $\left.\mathrm{m}, \mathrm{OCH}_{2}\right), 4.17-4.04\left(2 \mathrm{H}, \mathrm{m}, \mathrm{OCH}_{2}\right), 3.26-3.12\left(2 \mathrm{H}, \mathrm{m}, \mathrm{CHCH}_{2}\right), 1.32-0.98\left(6 \mathrm{H}, \mathrm{m}, 2 \mathrm{CH}_{3}\right) ;{ }^{13} \mathrm{C}$ NMR (75.5 MHz, $\left.\mathrm{CDCl}_{3}\right): \delta 180.01(\mathrm{C}=\mathrm{S}), 170.63(\mathrm{C}=\mathrm{O}), 169.97(\mathrm{C}=\mathrm{O}), 159.35(\mathrm{C}=\mathrm{N}, \mathrm{C} 2)$, $155.92(\mathrm{C}=\mathrm{N}, \mathrm{C} 4), 151.60\left(\mathrm{C}_{\mathrm{qAr}}\right), 137.12\left(\mathrm{C}_{\mathrm{qAr}}\right), 134.53\left(\mathrm{CH}_{\mathrm{Ar}}\right), 131.10\left(\mathrm{CH}_{\mathrm{Ar}}\right), 129.85\left(\mathrm{CH}_{\mathrm{Ar}}\right)$, $129.20\left(\mathrm{CH}_{\mathrm{Ar}}\right), 128.75\left(\mathrm{CH}_{\mathrm{Ar}}\right), 127.72\left(\mathrm{CH}_{\mathrm{Ar}}\right), 120.53\left(\mathrm{CH}_{\mathrm{Ar}}\right), 112.62\left(\mathrm{C}_{\mathrm{qAr}}\right), 62.34\left(\mathrm{OCH}_{2}\right)$, $61.19\left(\mathrm{OCH}_{2}\right), 55.87\left(\mathrm{CHCH}_{2}\right), 36.10\left(\mathrm{CHCH}_{2}\right), 14.30\left(\mathrm{CH}_{3}\right), 14.12\left(\mathrm{CH}_{3}\right)$. Anal. Calcd. for $\mathrm{C}_{23} \mathrm{H}_{24} \mathrm{~N}_{4} \mathrm{O}_{4} \mathrm{~S}$ (452.5): C, 61.05; H, 5.35; N, 12.38; S, 7.09. Found: C, 61.05; H, 5.33; N, 12.36; $\mathrm{S}, 7.05$.

Ethyl 2-phenyl-2-[3-(2-phenylquinazolin-4-yl)thioureido]acetate $\quad(5 d)$ From $d l$ $\mathrm{PhgOC}_{2} \mathrm{H}_{5} \cdot \mathrm{HCl}$ 2d (1.08 g). White crystals (1.43 g, 67\%); mp 157-158 ${ }^{\circ} \mathrm{C} .{ }^{1} \mathrm{H}$ NMR (300 $\left.\mathrm{MHz}_{\mathrm{CDCl}}\right): \delta 12.65\left(1 \mathrm{H}, \mathrm{d}, J=4.75 \mathrm{~Hz}, \mathrm{NHCH}_{2}\right), 8.94(1 \mathrm{H}, \mathrm{s}, 4-\mathrm{NH}), 8.27-8.25(2 \mathrm{H}, \mathrm{m}$, ArH), 8.05-8.02 (1H, m, ArH), 7.92-7.87 (2H, m, ArH), 7.64-7.36 (9H, m, ArH), 6.25 (1H, d, $J$ $=4.7 \mathrm{~Hz}, \mathrm{NHC} \underline{\mathrm{H}}), 4.40-4.19\left(2 \mathrm{H}, \mathrm{m}, \mathrm{OCH}_{2}\right), 1.27\left(3 \mathrm{H}, \mathrm{t}, J=6.9 \mathrm{~Hz}, \mathrm{CH}_{3}\right) ;{ }^{13} \mathrm{C} \mathrm{NMR}(75.5$ $\left.\mathrm{MHz}, \mathrm{CDCl}_{3}\right): \delta 180.11(\mathrm{C}=\mathrm{S}), 170.00(\mathrm{C}=\mathrm{O}), 158.91(\mathrm{C}=\mathrm{N}, \mathrm{C} 2), 155.93(\mathrm{C}=\mathrm{N}, \mathrm{C} 4), 151.43$ $\left(\mathrm{C}_{\mathrm{qAr}}\right), 135.57\left(\mathrm{C}_{\mathrm{qAr}}\right), 134.59\left(\mathrm{CH}_{\mathrm{Ar}}\right), 130.96\left(\mathrm{CH}_{\mathrm{Ar}}\right), 129.81\left(\mathrm{CH}_{\mathrm{Ar}}\right), 129.45\left(\mathrm{CH}_{\mathrm{Ar}}\right), 129.16$ $\left(\mathrm{CH}_{\mathrm{Ar}}\right), 128.81\left(\mathrm{CH}_{\mathrm{Ar}}\right), 128.62\left(\mathrm{CH}_{\mathrm{Ar}}\right), 128.23\left(\mathrm{CH}_{\mathrm{Ar}}\right), 127.80\left(\mathrm{CH}_{\mathrm{Ar}}\right), 120.56\left(\mathrm{CH}_{\mathrm{Ar}}\right), 112.62$ $\left(\mathrm{C}_{\mathrm{qAr}}\right), 63.33(\mathrm{CH}), 62.22\left(\mathrm{OCH}_{2}\right), 14.27\left(\mathrm{CH}_{2} \mathrm{CH}_{3}\right)$. Anal. Calcd. for $\mathrm{C}_{25} \mathrm{H}_{22} \mathrm{~N}_{4} \mathrm{O}_{2} \mathrm{~S}(442.5)$ : $\mathrm{C}$, 67.85; H, 5.01; N, 12.66; S, 7.24. Found: C, 67.83; H, 4.99; N, 12.54; S, 7.23.

Ethyl 3-[3-(2-phenylquinazolin-4-yl)thioureido]propanoate (5e). From $\beta-\mathrm{AlaOC}_{2} \mathrm{H}_{5} \cdot \mathrm{HCl} \mathbf{2 e}$ (0.77 g). White crystals (1.65 g, 90\%); mp 173-174 ${ }^{\circ} \mathrm{C} .{ }^{1} \mathrm{H}$ NMR $\left(300 \mathrm{MHz}, \mathrm{CDCl}_{3}\right): \delta 12.30$ $\left(1 \mathrm{H}, \mathrm{s}, \mathrm{NHCH}_{2}\right), 8.85(1 \mathrm{H}, \mathrm{s}, 4-\mathrm{NH}), 8.53-8.51(2 \mathrm{H}, \mathrm{m}, \mathrm{ArH}), 8.03-7.98(1 \mathrm{H}, \mathrm{m}, \mathrm{ArH}), 7.93-$ 7.89 (2H, m, ArH), 7.64-7.55 (4H, m, ArH), 4.16-4.10 (4H, m, NHC플, $\left.\mathrm{OCH}_{2}\right), 2.90(2 \mathrm{H}, \mathrm{t}, J=$ $\left.6.5 \mathrm{~Hz}, \mathrm{CH}_{2} \mathrm{CO}\right), 1.19\left(3 \mathrm{H}, \mathrm{t}, J=7.0 \mathrm{~Hz}, \mathrm{CH}_{3}\right) ;{ }^{13} \mathrm{C} \mathrm{NMR}\left(75.5 \mathrm{MHz}, \mathrm{CDCl}_{3}\right): \delta 180.48(\mathrm{C}=\mathrm{S})$, $171.99(\mathrm{C}=\mathrm{O}), 158.91(\mathrm{C}=\mathrm{N}, \mathrm{C} 2), 155.95(\mathrm{C}=\mathrm{N}, \mathrm{C} 4), 151.52\left(\mathrm{C}_{\mathrm{qAr}}\right), 137.36\left(\mathrm{C}_{\mathrm{qAr}}\right), 134.54$ $\left(\mathrm{CH}_{\mathrm{Ar}}\right), 131.14\left(\mathrm{CH}_{\mathrm{Ar}}\right), 129.81\left(\mathrm{CH}_{\mathrm{Ar}}\right), 128.98\left(\mathrm{CH}_{\mathrm{Ar}}\right), 128.60\left(\mathrm{CH}_{\mathrm{Ar}}\right), 127.73\left(\mathrm{CH}_{\mathrm{Ar}}\right), 120.60$ $\left(\mathrm{CH}_{\mathrm{Ar}}\right), 112.58\left(\mathrm{C}_{\mathrm{qAr}}\right), 61.01\left(\mathrm{OCH}_{2}\right), 41.871\left(\mathrm{NCH}_{2}\right), 33.27\left(\mathrm{COCH}_{2}\right), 14.35\left(\mathrm{CH}_{3}\right)$. Anal. 
Calcd. for $\mathrm{C}_{20} \mathrm{H}_{20} \mathrm{~N}_{4} \mathrm{O}_{2} \mathrm{~S}$ (380.5): C, 63.14; H, 5.30; N, 14.73; S, 8.43. Found: 63.06; H, 5.27; N, 14.68; S, 8.41.

\section{Derivatization reactions of 5 a}

Ethyl 2-(3-(2-phenylquinazolin-4-yl)thioureido)acetate (5f). To a stirred solution of 5a (1.762 $\mathrm{g}, 5 \mathrm{mmol})$ in absolute ethanol was added $\mathrm{KOH}(0.28 \mathrm{~g}, 5 \mathrm{mmol})$. The reaction mixture was refluxed for $4 \mathrm{~h}$, and then evaporated under reduced pressure. The residue was collected and crystallized from ethanol to give white crystals $\mathbf{5 f}(1.23 \mathrm{~g}, 69 \%)$; mp 204-205 ${ }^{\circ} \mathrm{C}$. ${ }^{1} \mathrm{H}$ NMR (300 $\left.\mathrm{MHz}, \mathrm{CDCl}_{3}\right): \delta 12.30\left(1 \mathrm{H}, \mathrm{s}, \mathrm{NHCH}_{2}\right), 8.93(1 \mathrm{H}, \mathrm{s}, 4-\mathrm{NH}), 8.54-8.51(2 \mathrm{H}, \mathrm{m}, \mathrm{ArH}), 8.04-8.01$ $(1 \mathrm{H}, \mathrm{m}, \mathrm{ArH}), 7.93-7.88(2 \mathrm{H}, \mathrm{m}, \mathrm{ArH}), 7.64-7.56(4 \mathrm{H}, \mathrm{m}, \mathrm{ArH}), 4.68(2 \mathrm{H}, \mathrm{d}, J=4.7 \mathrm{~Hz}$, $\left.\mathrm{NHCH}_{2}\right), 4.33\left(2 \mathrm{H}, \mathrm{q}, J=6.8 \mathrm{~Hz}, \mathrm{OCH}_{2}\right) ; 1.32\left(3 \mathrm{H}, \mathrm{t}, J=6.9 \mathrm{~Hz}, \mathrm{CH}_{3}\right)$. Anal. Calcd. for $\mathrm{C}_{19} \mathrm{H}_{18} \mathrm{~N}_{4} \mathrm{O}_{2} \mathrm{~S}$ (366.4): C, 62.28; H, 4.95; N, 15.29; S, 8.75. Found: C, 62.11; H, 4.93; N, 15.18; S, 8.74.

Reactions with alkyl halides. To a stirred mixture of $5 \mathbf{a}(1.762 \mathrm{~g}, 5 \mathrm{mmol})$ in DMF (20 mL) and triethyl amine $(0.7 \mathrm{~mL}, 5 \mathrm{mmol})$ was added the appropriate alkyl halide $(5 \mathrm{mmol})$. The reaction mixture was heated at $90{ }^{\circ} \mathrm{C}$ for $4 \mathrm{~h}$, and was then evaporated under reduced pressure. The residue was recrystallized from ethanol.

Methyl 2-[2-methyl-1-(2-phenylquinazolin-4-yl)isothioureido]acetate (6). Using methyl iodide $(0.30 \mathrm{~mL})$. White crystals $(1.27 \mathrm{~g}, 69 \%) ; \mathrm{mp} 176-177^{\circ} \mathrm{C} .{ }^{1} \mathrm{H} \mathrm{NMR}\left(300 \mathrm{MHz}, \mathrm{CDCl}_{3}\right): \delta$ $12.60\left(1 \mathrm{H}, \mathrm{s}, \mathrm{NHCH}_{2}\right), 8.55-8.51(2 \mathrm{H}, \mathrm{m}, \mathrm{ArH}), 7.93-7.88(3 \mathrm{H}, \mathrm{m}, \mathrm{ArH}), 7.59-7.47(4 \mathrm{H}, \mathrm{m}$, $\mathrm{ArH}), 4.34\left(2 \mathrm{H}, \mathrm{s}, \mathrm{NCH}_{2}\right), 3.43\left(3 \mathrm{H}, \mathrm{s}, \mathrm{OCH}_{3}\right), 2.74\left(3 \mathrm{H}, \mathrm{s}, \mathrm{SCH}_{3}\right)$. Anal. Calcd. for $\mathrm{C}_{19} \mathrm{H}_{18} \mathrm{~N}_{4} \mathrm{O}_{2} \mathrm{~S}$ (366.4): C, 62.28; H, 4.95; N, 15.29; S, 8.75. Found: C, 62.23; H, 4.91; N, 15.22; $\mathrm{S}, 8.70$.

Methyl [4-oxo-2-(2-phenylquinazolin-4-ylimino)thiazolidin-3-yl]acetate (7). From methyl chloroacetate $(0.45 \mathrm{~mL})$ : White crystals $(1.32 \mathrm{~g}, 67 \%)$; mp $185-186{ }^{\circ} \mathrm{C} .{ }^{1} \mathrm{H} \mathrm{NMR}(300 \mathrm{MHz}$, $\left.\mathrm{CDCl}_{3}\right): \delta 8.63(2 \mathrm{H}, \mathrm{d}, J=7.7 \mathrm{~Hz}, \mathrm{ArH}), 8.36(1 \mathrm{H}, \mathrm{d}, J=8.0 \mathrm{~Hz}, \mathrm{ArH}), 8.04(1 \mathrm{H}, \mathrm{d}, J=8.4 \mathrm{~Hz}$, $\mathrm{ArH}), 7.89-7.84(1 \mathrm{H}, \mathrm{m}, \mathrm{ArH}), 7.55-7.53(4 \mathrm{H}, \mathrm{m}, \mathrm{ArH}), 4.81\left(2 \mathrm{H}, \mathrm{s}, \mathrm{NCH}_{2}\right), 3.99(2 \mathrm{H}, \mathrm{s}$, $\left.\mathrm{SCH}_{2}\right), 3.82\left(3 \mathrm{H}, \mathrm{s}, \mathrm{OCH}_{3}\right) ;{ }^{13} \mathrm{C} \mathrm{NMR}\left(75.5 \mathrm{MHz}, \mathrm{CDCl}_{3}\right): \delta 171.82(\mathrm{C}=\mathrm{O}), 167.59(\mathrm{C}=\mathrm{O})$, $160.17\left(\mathrm{C}_{\mathrm{qAr}}\right), 152.70\left(\mathrm{C}_{\mathrm{qAr}}\right), 139.36\left(\mathrm{C}_{\mathrm{qAr}}\right), 138.38\left(\mathrm{CH}_{\mathrm{Ar}}\right), 137.31\left(\mathrm{C}_{\mathrm{qAr}}\right), 134.10\left(\mathrm{CH}_{\mathrm{Ar}}\right), 130.74$ $\left(\mathrm{CH}_{\mathrm{Ar}}\right), 128.75\left(\mathrm{CH}_{\mathrm{Ar}}\right), 128.43\left(\mathrm{CH}_{\mathrm{Ar}}\right), 126.92\left(\mathrm{CH}_{\mathrm{Ar}}\right), 125.15\left(\mathrm{CH}_{\mathrm{Ar}}\right), 119.26\left(\mathrm{C}_{\mathrm{qAr}}\right), 52.95$ $\left(\mathrm{OCH}_{3}\right), 44.50\left(\mathrm{NCH}_{2}\right), 34.19\left(\mathrm{SCH}_{2}\right)$. Anal. Calcd. for $\mathrm{C}_{20} \mathrm{H}_{16} \mathrm{~N}_{4} \mathrm{O}_{3} \mathrm{~S}$ (392.4): C, 61.21; H, 4.11; N, 14.28; S, 8.17. Found: C, 61.16; H, 4.08; N, 14.15; S, 8.13.

Methyl [4-hydroxy-4-phenyl-2-(2-phenylquinazolin-4-ylimino)thiazolidin-3-yl]acetate (8a). From phenacyl bromide (1.0 g). White crystals $(1.68 \mathrm{~g}, 71 \%)$; mp $208-209{ }^{\circ} \mathrm{C} .{ }^{1} \mathrm{H}$ NMR $(300$ $\left.\mathrm{MHz}, \mathrm{CDCl}_{3}\right): \delta 8.59-8.51(2 \mathrm{H}, \mathrm{m}, \mathrm{ArH}), 8.37(1 \mathrm{H}, \mathrm{d}, J=8.1 \mathrm{~Hz}, \mathrm{ArH}), 8.03(1 \mathrm{H}, \mathrm{d}, J=8.2 \mathrm{~Hz}$, ArH), 7.83-7.81 (1H, m, ArH), 7.71-7.68 (2H, m, ArH), 7.53-7.34 (7H, m, ArH), 5.85 (1H, bs, $\mathrm{OH}), 4.38\left(1 \mathrm{H}, \mathrm{d}, J=16.8 \mathrm{~Hz}, \mathrm{NCH}_{\mathrm{A}}\right), 3.74\left(3 \mathrm{H}, \mathrm{s}, \mathrm{OCH}_{3}\right), 3.60\left(1 \mathrm{H}, \mathrm{d}, J=11.9 \mathrm{~Hz}, \mathrm{SCH}_{\mathrm{A}}\right)$, $3.49\left(2 \mathrm{H}, \mathrm{d}, J=11.7 \mathrm{~Hz}, \mathrm{NCH}_{\mathrm{B}}, \mathrm{SCH}_{\mathrm{B}}\right) ;{ }^{13} \mathrm{C} \mathrm{NMR}\left(75.5 \mathrm{MHz}, \mathrm{CDCl}_{3}\right): \delta 171.82\left(\mathrm{C}_{\mathrm{qAr}}\right), 165.85$ $\left(\mathrm{C}_{\mathrm{qAr}}\right), 163.46\left(\mathrm{C}_{\mathrm{qAr}}\right), 160.73\left(\mathrm{C}_{\mathrm{qAr}}\right), 152.17\left(\mathrm{C}_{\mathrm{qAr}}\right), 141.01\left(\mathrm{C}_{\mathrm{qAr}}\right), 138.69\left(\mathrm{C}_{\mathrm{qAr}}\right), 133.41\left(\mathrm{CH}_{\mathrm{Ar}}\right)$, $130.53\left(\mathrm{CH}_{\mathrm{Ar}}\right), 129.40\left(\mathrm{CH}_{\mathrm{Ar}}\right), 129.13\left(\mathrm{CH}_{\mathrm{Ar}}\right), 128.98\left(\mathrm{CH}_{\mathrm{Ar}}\right), 128.55\left(\mathrm{CH}_{\mathrm{Ar}}\right), 127.60\left(\mathrm{CH}_{\mathrm{Ar}}\right)$, $126.91\left(\mathrm{CH}_{\mathrm{Ar}}\right), 126.13\left(\mathrm{CH}_{\mathrm{Ar}}\right), 125.69\left(\mathrm{CH}_{\mathrm{Ar}}\right), 121.00\left(\mathrm{C}_{\mathrm{qAr}}\right), 92.74(\mathrm{COH}), 52.68\left(\mathrm{OCH}_{3}\right), 47.16$ 
$\left(\mathrm{NCH}_{2}\right), 45.54\left(\mathrm{SCH}_{2}\right)$. Anal. Calcd. for $\mathrm{C}_{26} \mathrm{H}_{22} \mathrm{~N}_{4} \mathrm{O}_{3} \mathrm{~S}$ (470.5): C, 66.37; H, 4.71; N, 11.91; S, 6.81. Found: C, 66.29; H, 4.70; N, 11.88; S, 6.79.

Methyl [4-hydroxy-2-(2-phenylquinazolin-4-ylimino)-4-tolylthiazolidin-3-yl]acetate (8b). From 4-methylphenacyl bromide $(1.07 \mathrm{~g})$ : White crystals $(1.45 \mathrm{~g}, 60 \%)$; mp $194-195{ }^{\circ} \mathrm{C} .{ }^{1} \mathrm{H}$ NMR (300 MHz, $\left.\mathrm{CDCl}_{3}\right): \delta 8.60-8.53(2 \mathrm{H}, \mathrm{m}, \mathrm{ArH}), 8.37(1 \mathrm{H}, \mathrm{d}, J=8.0 \mathrm{~Hz}, \mathrm{ArH}), 8.02(1 \mathrm{H}, \mathrm{d}$, $J=8.0 \mathrm{~Hz}, \mathrm{ArH}), 7.83-7.80(1 \mathrm{H}, \mathrm{m}, \mathrm{ArH}), 7.68-7.59$ (6H, m, ArH), 7.35-7.17 (2H, m, ArH), $5.70(1 \mathrm{H}, \mathrm{bs}, \mathrm{OH}), 4.43\left(1 \mathrm{H}, \mathrm{d}, J=16.9 \mathrm{~Hz}, \mathrm{NCH}_{\mathrm{A}}\right), 3.74\left(3 \mathrm{H}, \mathrm{s}, \mathrm{OCH}_{3}\right), 3.59(1 \mathrm{H}, \mathrm{d}, J=11.9$ $\left.\mathrm{Hz}, \mathrm{SCH}_{\mathrm{A}}\right), 3.52\left(2 \mathrm{H}, \mathrm{d}, J=11.9 \mathrm{~Hz}, \mathrm{NCH}_{\mathrm{B}}, \mathrm{SCH}_{\mathrm{B}}\right), 2.41\left(3 \mathrm{H}, \mathrm{s}, \mathrm{CH}_{3}\right) ;{ }^{13} \mathrm{C} \mathrm{NMR}(75.5 \mathrm{MHz}$, $\left.\mathrm{CDCl}_{3}\right): \delta 172.22(\mathrm{C}=\mathrm{O}), 165.85\left(\mathrm{C}_{\mathrm{qAr}}\right), 163.46\left(\mathrm{C}_{\mathrm{qAr}}\right), 160.73\left(\mathrm{C}_{\mathrm{qAr}}\right), 152.17\left(\mathrm{C}_{\mathrm{qAr}}\right), 141.01$ $\left(\mathrm{C}_{\mathrm{qAr}}\right), 139.15\left(\mathrm{C}_{\mathrm{qAr}}\right), 133.41\left(\mathrm{CH}_{\mathrm{Ar}}\right), 130.50\left(\mathrm{CH}_{\mathrm{Ar}}\right), 129.72\left(\mathrm{CH}_{\mathrm{Ar}}\right), 129.25\left(\mathrm{CH}_{\mathrm{Ar}}\right), 128.58$ $\left(\mathrm{CH}_{\mathrm{Ar}}\right), 127.82\left(\mathrm{CH}_{\mathrm{Ar}}\right), 126.81\left(\mathrm{CH}_{\mathrm{Ar}}\right), 126.13\left(\mathrm{CH}_{\mathrm{Ar}}\right), 125.50\left(\mathrm{CH}_{\mathrm{Ar}}\right), 120.89\left(\mathrm{C}_{\mathrm{qAr}}\right), 92.83$ $(\mathrm{COH}), 52.95\left(\mathrm{OCH}_{3}\right), 47.34\left(\mathrm{NCH}_{2}\right), 45.53\left(\mathrm{SCH}_{2}\right), 21.37\left(\mathrm{CH}_{3}\right)$. Anal. Calcd. for $\mathrm{C}_{27} \mathrm{H}_{24} \mathrm{~N}_{4} \mathrm{O}_{3} \mathrm{~S}$ (484.6): C, 66.92; H, 4.99; N, 11.56; S, 6.62. Found: C, 66.87; H, 4.91; N, 11.43; S, 6.52.

Methyl [4-hydroxy-4-(4-methoxyphenyl)-2-(2-phenylquinazolin-4-ylimino)thiazolidin-3-yl]acetate (8c). From 4-methoxyphenacyl bromide $(1.15 \mathrm{~g})$ : Yellowish white crystals (1.54 g, 64\%); mp 198-199 ${ }^{\circ} \mathrm{C} .{ }^{1} \mathrm{H}$ NMR $\left(300 \mathrm{MHz}, \mathrm{CDCl}_{3}\right)$ : $\delta 8.61-8.59(2 \mathrm{H}, \mathrm{m}, \mathrm{ArH}), 8.36(1 \mathrm{H}, \mathrm{d}, J=$ $8.0 \mathrm{~Hz}, \mathrm{ArH}), 8.01(1 \mathrm{H}, \mathrm{d}, J=8.4 \mathrm{~Hz}, \mathrm{ArH}), 7.84-7.80(1 \mathrm{H}, \mathrm{m}, \mathrm{ArH}), 7.62(2 \mathrm{H}, \mathrm{d}, J=8.8 \mathrm{~Hz}$, ArH), 7.55-7.46 (4H, m, ArH), 6.97 (2H, d, $J=8.8 \mathrm{~Hz}, \mathrm{ArH}), 5.76$ (1H, bs, OH), 4.39 (1H, d, $J$ $\left.=16.9 \mathrm{~Hz}, \mathrm{NCH}_{\mathrm{A}}\right), 3.86\left(3 \mathrm{H}, \mathrm{s}, \mathrm{OCH}_{3}\right), 3.82\left(1 \mathrm{H}, \mathrm{d}, J=11.9 \mathrm{~Hz}, \mathrm{SCH}_{\mathrm{A}}\right), 3.74\left(3 \mathrm{H}, \mathrm{s}, \mathrm{OCH}_{3}\right)$, $3.58\left(1 \mathrm{H}, \mathrm{d}, J=16.8 \mathrm{~Hz}, \mathrm{NCH}_{\mathrm{B}}\right), 3.49\left(1 \mathrm{H}, \mathrm{d}, J=12.1 \mathrm{~Hz}, \mathrm{SCH}_{\mathrm{B}}\right) ;{ }^{13} \mathrm{C} \mathrm{NMR}(75.5 \mathrm{MHz}$, $\left.\mathrm{CDCl}_{3}\right): \delta 171.89(\mathrm{C}=\mathrm{O}), 166.06\left(\mathrm{C}_{\mathrm{qAr}}\right), 163.46\left(\mathrm{C}_{\mathrm{qAr}}\right), 161.15\left(\mathrm{C}_{\mathrm{qAr}}\right), 152.14\left(\mathrm{C}_{\mathrm{qAr}}\right), 139.15$ $\left(\mathrm{C}_{\mathrm{qAr}}\right), 132.85\left(\mathrm{CH}_{\mathrm{Ar}}\right), 130.49\left(\mathrm{CH}_{\mathrm{Ar}}\right), 129.69\left(\mathrm{CH}_{\mathrm{Ar}}\right), 129.25\left(\mathrm{CH}_{\mathrm{Ar}}\right), 128.27\left(\mathrm{CH}_{\mathrm{Ar}}\right), 127.81$ $\left(\mathrm{CH}_{\mathrm{Ar}}\right), 126.14\left(\mathrm{CH}_{\mathrm{Ar}}\right), 125.50\left(\mathrm{CH}_{\mathrm{Ar}}\right), 120.55\left(\mathrm{C}_{\mathrm{qAr}}\right), 92.97(\mathrm{COH}), 55.78\left(\mathrm{OCH}_{3}\right), 53.18$ $\left(\mathrm{OCH}_{3}\right), 47.47\left(\mathrm{NCH}_{2}\right), 46.14\left(\mathrm{SCH}_{2}\right)$. Anal. Calcd. For $\mathrm{C}_{27} \mathrm{H}_{24} \mathrm{~N}_{4} \mathrm{O}_{4} \mathrm{~S}$ (500.57): C, 64.78; H, 4.83; N, 11.19; S, 6.41. Found: C, 64.74; H, 4.78; N, 11.12; S, 6.41.

\section{References}

1. Ahgren, C.; Backro, K.; Bell, F.W.; Cantrell, A. S.; Clemens, M.; Colacino, J. ; Deeter, M. J. B.; Engelhardt, J. A.; Jaskunas, S. R.; Johansson, N. G.; Jordan, C L.; Kasher, J. S.; Kinnick, M. D.; Lind, P.; Lopez, C.; Morin, J. M.; Muesing, M. A.; Noreen, R.; Oberg, B.; Paget, C. J.; Palkowitz, J. A.; Parrish, C.; Pranc, P.; Rippy, M. K.; Rydergard, C.; Sahlberg, C.; Swanson, S.; Ternansky, R.; Unge, J.; Vasileff, T. R. T.; Vrang, L.; West, S. J.; Zhang, H.; Zhou, X. X. Antimicrob. Agents Chemoth. 1995, 39, 1329.

2. Heinisch, G.; Matuszczak, B.; Pachler, S.; Rakowitz, D. Antivir. Chem. Chemother. 1997, 8, 443.

3. Ren, J.; Diprose, J.; Warren, J.; Esnouf, R. M.; Bird, L. E.; Ikemizu, S.; Slater, M.; Milton, J.; Balzarini, J.; Stuart, D. I; Stammers, D. K. J. Biol. Chem. 2000, 275, 5633. 
4. Ahgren, C.; Backro, K.; Bell, F. W.; Cantrell, S.; Clemens, M.; Colacino, J. M.; Deeter, M. J. B.; Engelhardt, J. A.; Hogberg, M.; Jaskunas, S. R. Antimicrob. Agents Chemoth. 1995, 39, 1329.

5. Venkatachalam, T. K.; Uckun, F. M. Synth. Commun. 2006, 36, 2103.

6. Venkatachalam, T. K.; Sudbeck, E.; Uckun, F. M. J. Mol. Struc. 2004, 687, 45.

7. Venkatachalam, T. K.; Qazi, B. S.; Samuel, P.; Uckun, F. M. Bioorganic \& Medicinal Chemistry 2003, 11, 1095.

8. Fathalla, W.; Cajan, M.; Marek, J.; Pazdera, P. Molecules 2001, 6, 574.

9. Fathalla, W.; Cajan, M.; Marek, J.; Pazdera, P. Molecules 2001, 6, 588.

10. Fathalla, W.; Pazdera, P. Arkivoc 2002, 1, 7.

11. Szczepur, L. F.; Eilts, K. K.; Hermetet, A. K.; Ackerman, L. J.; Sweingen, J. K.; West, D. X. J. Mol. Struc. 2002, 607, 101.

12. West, D. X.; Swearingen, J. K.; Hermetet, A. K.; Ackerman, L. J. Mol. Struc. 2001, 562, 95.

13. Fathalla, W.; Cajan, M.; Pazdera, P. Molecules 2001, 6, 557.

14. Mohareb, R. M.; Sherif, M. Heteroatom Chem. 1997, 8, 77. 